\section{Investigación de vectores y reservorios en brote de Chagas agudo por posible transmisión oral en Aguachica, Cesar, Colombia}

\author{
Investigation of vectors and reservoirs in an acute \\ Chagas outbreak due to possible oral transmission \\ in Aguachica, Cesar, Colombia
}

\author{
Investigação dos vetores e reservatórios ao \\ surto da doença de Chagas agudo por possível \\ transmissão oral em Aguachica, Cesar, Colômbia
}

\author{
Hugo Soto 1 \\ Tania Tibaduiza 2 \\ Marleny Montilla 2 \\ Omar Triana ${ }^{3}$ \\ Diana Carolina Suárez 4 \\ Mariela Torres Torres 2 \\ María Teresa Arias 1 \\ Ligia Lugo 1
}

\author{
1 Secretaría Departamental \\ de Salud del Cesar, \\ Valledupar, Colombia. \\ 2 Instituto Nacional de Salud, \\ Bogotá, Colombia. \\ 3 Universidad de Antioquia, \\ Bucaramanga, Colombia. \\ ${ }^{4}$ Universidad Industrial de \\ Santander, Bucaramanga, \\ Colombia. \\ Correspondencia \\ M. T. Torres \\ Instituto Nacional de Salud. \\ Avenida Calle 26 51-20 Zona \\ Postal 6 CAN, Bogotá DCl \\ Cundinamarca-Distrito \\ Capital, Colombia. \\ mtorres@ins.gov.co
}

\begin{abstract}
Colombia recorded 11 cases of acute Chagas disease and 80 cases of oral contamination with Trypanosoma cruzi. The current study analyzes the entomological and parasitological characteristics of the outbreak in Aguachica, Cesar Department, in 2010. An interdisciplinary group of health professionals and regional university personnel conducted the laboratory tests in the patients and the investigation of the transmission focus. Eleven cases of acute Chagas diseases were detected in a single family in a dwelling with domiciliated triatomines and Rhodnius pallescens, Pantrongylus geniculatus, Eratyrus cuspidatus, and two Didelphis marsupialis opossums infected with T. cruzi in Attalea butyracea and Elaeis oleifera palm trees in the urban area of Aguachica. The study analyzes the role of R. pallescens and palm trees in the wild cycle of $\mathrm{T}$. cruzi and in oral transmission of Chagas disease. Sporadic incursions by wild R. pallescens, P. geniculatus, and $\mathrm{E}$. cuspidatus from the nearby palm trees into human dwellings may cause increasingly frequent outbreaks of oral Chagas disease.
\end{abstract}

Chagas Disease; Trypanosoma cruzi; Disease Outbreaks

\section{Resumen}

Colombia tiene un registro de 11 casos de Chagas agudo y 80 casos por contaminación oral con Trypanosoma cruzi. Este trabajo analiza los hallazgos entomológicos y parasitológicos del brote de Aguachica, Cesar, en 2010. Un grupo interdisciplinario de profesionales de la salud y de universidades regionales realizó las pruebas de laboratorio a los pacientes y el estudio del foco de transmisión. Se detectaron 11 casos agudos de enfermedad de Chagas en una sola familia con vivienda sin triatominos domiciliados $y$, Rhodnius pallescens, Pantrongylus geniculatus, Eratyrus cuspidatus y dos Didelphis marsupialis infectados con T. cruzi en palmas de Attalea butyracea y Elaeis oleifera del área urbana de Aguachica. Se analiza la participación del R. pallescens y el rol de las palmas en el ciclo silvestre de T. cruzi y para la transmisión oral de la enfermedad de Chagas. Incursiones esporádicas de R. pallescens, P. geniculatus y E. cuspidatus silvestres desde palmas cercanas al domicilio humano pueden provocar brotes cada vez más frecuentes de Chagas oral.

Enfermedad de Chagas; Trypanosoma cruzi; Brotes de Enfermedades 


\section{Introducción}

La enfermedad de Chagas constituye un importante problema de salud pública en el continente americano. Esta enfermedad es causada por el parásito Trypanosoma cruzi y forma parte de un grupo de enfermedades que afectan a poblaciones vulnerables 1 , con aproximadamente 7,7 millones de infectados y 70 millones en riesgo de infección en 22 países de América Latina, lo que equivale a un $27 \%$ de prevalencia (1.448 individuos infectados por 100.000 habitantes) y una tasa de incidencia anual de 0,008 casos por 100.000 habitantes, causando cerca de 15.000 muertes cada año en el continente. En la fase aguda los decesos ocurren mayormente en niños de hasta tres años 2.

En Colombia, un 5\% de la población que vive en zonas endémicas está infectada, lo que equivale a 700.000 individuos distribuidos en Arauca $(21,1 \%)$, Casanare $(10 \%)$, Santander $(6,3 \%)$, Norte de Santander $(5,2 \%)$, Boyacá $(3,7 \%)$, Cundinamarca $(1,9 \%)$ y Meta $(1,7 \%) 3,4$; el número de infecciones pasó de 900.000 en 1995 a 436.000 en 2005 y la incidencia pasó de 39.162 en 1990, a 3.133 en 1995, y 5.250 en $2005^{3}$.

El T. cruzi es mayormente transmitido a los humanos por insectos hematófagos de la subfamilia Triatominae (Hemiptera: Reduviidae). La transmisión de la infección también ocurre por la placenta, vía transfusión sanguínea, trasplante de órganos, accidentes de laboratorio, o por ingestión de alimentos o bebidas contaminados con triatominos o heces infectadas con T. cruzi 4 . No obstante, la perennidad de la transmisión antropo-zoonótica implica que casos humanos ocurran de forma esporádica o eventual por diversos mecanismos, como la intrusión de vectores en el domicilio o por su actividad en el extradomicilio, ambos asociados a los ciclos silvestres, sin colonización del domicilio por los vectores. La transmisión oral en su origen está también relacionada con los focos selváticos o con elementos que componen la cadena de transmisión en el ambiente natural 5 . En este sentido, la enfermedad de Chagas es una zoonosis donde los ciclos naturales de transmisión del parásito estarán siempre presentes, su erradicación es casi imposible y el riesgo de transmisión al hombre siempre tendrá una probabilidad 6,7.

Durante los últimos años, de los más de 1.000 casos agudos de la enfermedad de Chagas reportados en 138 brotes en la cuenca amazónica, 776 han sido atribuidos a la ingestión de alimentos contaminados con heces de triatominos infectadas con T. $_{\text {cruzi }}{ }^{8}$. La enfermedad de Chagas oral, por la ingestión de alimentos mezclados con triatominos o con sus heces infectadas con
T. cruzi, está bien documentada en la Amazonía brasileña 9,10,11,12. Igualmente, la literatura evidencia la supervivencia de T. cruzi en bebidas por más de 24 horas post-contaminación 13,14, lo que significa una ventana de incubación in vitro que puede permitir la transmisión de $T$. cruzi por vía oral $15,16,17$.

La transmisión oral de la enfermedad de Chagas presenta una tendencia al incremento, documentada en diversos brotes agudos por posible transmisión oral en Brasil, Colombia, México y Venezuela. Esto se ha evidenciado en parte después del relativo éxito del control de la trasmisión vectorial en estos países 18,19. En Colombia desde 1992 se han reportado 11 brotes de Chagas agudo por posible transmisión oral. En todos los brotes los casos corresponden a grupos de personas relacionados en tiempo y espacio, durante una reunión en la que se compartieron alimentos y bebidas preparados de forma doméstica y artesanal; en ninguno de los casos se presentaron señales de puerta de entrada 20 (Tabla 1). Paralelamente, en los estudios de campo se han logrado identificar reservorios marsupiales (Didelphidae) en los alrededores de las casas y triatominos silvestres de las especies Triatoma dimidiata, Panstrongylus geniculatus, y Rhodnius pallescens, ingresando en los domicilios o ubicados en los alrededores del mismo o inclusive en zonas urbanas 21 .

En el presente trabajo se describen las características ecoepidemiológicas y los hallazgos entomológicos, parasitológicos y de reservorios en el brote de Chagas agudo por posible transmisión oral, ocurrido en el municipio de Aguachica, departamento del Cesar, Colombia, en mayo de 2010, donde con anterioridad se tenía registro de dos especies de triatominos y 16 casos aislados de enfermedad de Chagas por bancos de sangre.

\section{Metodología}

\section{Área de estudio}

El municipio de Aguachica está ubicado al sur del departamento del Cesar, a $8^{\circ} 18^{\prime} 45^{\prime \prime}$ de latitud norte y $73^{\circ} 37^{\prime} 37^{\prime \prime}$ de longitud oeste del meridiano de Greenwich, entre la Cordillera Oriental y el valle del río Magdalena, a una distancia de $301 \mathrm{~km}$ de Valledupar, la capital del departamento. El municipio cuenta con una población urbana de 90.039 habitantes 22. El casco urbano presenta un relieve semiquebrado, cuya formación vegetal corresponde a bosque seco tropical (BsT, según la clasificación de Holdridge 23,24 , su altitud está entre 50 y 200msnm, y su temperatura media está en $28^{\circ} \mathrm{C}$. y precipitaciones entre los 700 y $2000 \mathrm{~mm}$. anuales, con uno o dos periodos 
Tabla 1

Brotes de Chagas agudo por posible transmisión oral en Colombia.

\begin{tabular}{|c|c|c|c|c|c|}
\hline & $\begin{array}{c}\text { Fecha de } \\
\text { notificación }\end{array}$ & $\begin{array}{c}\text { Municipio } \\
\text { (Departamento) }\end{array}$ & $\begin{array}{c}\text { Muertes/Casos } \\
\text { confirmados }\end{array}$ & $\begin{array}{l}\text { Vectores } \\
\text { asociados }\end{array}$ & $\begin{array}{l}\text { Reservorios } \\
\text { encontrados }\end{array}$ \\
\hline 1 & 1992 & $\begin{array}{l}\text { Tibú (Norte de } \\
\text { Santander) }\end{array}$ & $0 / 14$ & SD & SD \\
\hline 2 & 1999 & $\begin{array}{c}\text { Guamal } \\
\text { (Magdalena) }\end{array}$ & $5 / 13$ & SD & SD \\
\hline 3 & 2003 & $\begin{array}{l}\text { Bucaramanga } \\
\text { (Santander) }\end{array}$ & $3 / ?$ & SD & SD \\
\hline 4 & 06/Dic/2008 & Lebrija (Santander) & $2 / 10$ & $\begin{array}{l}\text { Panstrongylus } \\
\text { geniculatus }\end{array}$ & $\begin{array}{l}\text { Didelphis } \\
\text { marsupialis }\end{array}$ \\
\hline 5 & 18/Feb/2009 & $\begin{array}{c}\text { Bucaramanga } \\
\text { (Santander) }\end{array}$ & $1 / 5$ & $\begin{array}{c}\text { Panstrongylus } \\
\text { geniculatus, } \\
\text { Rhodnius } \\
\text { pallescens }\end{array}$ & $\begin{array}{l}\text { Didelphis } \\
\text { marsupialis }\end{array}$ \\
\hline 6 & 03/Abr/2009 & Villanueva (Bolívar) & $2 / 5$ & SD & SD \\
\hline 7 & 29/May/2009 & $\begin{array}{c}\text { Piedecuesta/Girón } \\
\text { (Santander) }\end{array}$ & $0 / 5$ & $\begin{array}{l}\text { Panstrongylus } \\
\text { geniculatus }\end{array}$ & $\begin{array}{l}\text { Didelphis } \\
\text { marsupialis }\end{array}$ \\
\hline 8 & May/2009 & $\begin{array}{c}\text { Sabana de Torres } \\
\text { (Santander) }\end{array}$ & $0 / 1$ & SD & $\mathrm{SD}$ \\
\hline 9 & 20/Ene/2010 & Turbo (Antioquia) & $1 / 11$ & $\begin{array}{l}\text { Panstrongylus } \\
\text { geniculatus }\end{array}$ & Caluromys lanatus \\
\hline 10 & 20/Feb/2010 & $\begin{array}{c}\text { San Vicente de } \\
\text { Chucurí (Santander) }\end{array}$ & $0 / 3$ & $\begin{array}{c}\text { Panstrongylus } \\
\text { geniculatus, } \\
\text { Rhodnius } \\
\text { pallescens }\end{array}$ & $\begin{array}{l}\text { Didelphis } \\
\text { marsupialis }\end{array}$ \\
\hline 11 & May/2010 & Girón (Santander) & $0 / 5$ & SD & $\begin{array}{l}\text { Didelphis } \\
\text { marsupialis }\end{array}$ \\
\hline
\end{tabular}

SD: sin datos.

Nota: el registro de eventos de Chagas agudo aumentó considerablemente durante la década del 2010.

Fuente: Instituto Nacional de Salud 55.

marcados de sequía al año 25 . En el área de estudio a $140 \mathrm{msnm}$. se observa abundante bosque secundario.

\section{Descripción del brote}

La Secretaría de Salud de Santander notificó el día 3 de junio de 2010 la ocurrencia de un brote de Chagas agudo en un grupo de personas familiares procedentes del municipio de Aguachica. De 11 casos confirmados, siete fueron identificados por consulta al Hospital Universitario de Santander (HUS) y los cuatro restantes identificados por búsqueda activa en la zona del brote. La sintomatología presentada por los casos fue fiebre prolongada (15 días a un mes de evolución), malestar general, osteomialgias, dolor abdominal, edema facial y en miembros inferiores y disnea de medianos a pequeños esfuerzos.
Un grupo interdisciplinario de funcionarios de la Secretaría de Salud del Cesar, la Secretaría municipal de Aguachica y del Instituto Nacional de Salud se desplazó al municipio para realizar la investigación ecoepidemiológica de campo.

\section{Diagnóstico parasitológico y serológico de los pacientes}

Los pacientes procedentes de Aguachica fueron diagnosticados por medios parasitológicos como el método microStrout para lo cual se centrifugó en un capilar de $5 \mu \mathrm{L}$ de la sangre de cada paciente y se buscaron bajo el microscopio los parásitos en el sobrenadante -fraccíon no celular. También se aplicaron métodos serológicos como ELISA e Inmunofluoresciencia Indirecta (IFI), cuyas concentraciones de corte aparecen al pie de la tabla de resultados según el labora- 
torio que los realizó 26. Adicionalmente, se realizó el hemocultivo en medios Tobie y LIT a las muestras de sangre de 53 personas contactos y convivientes de los casos confirmados.

Para el aislamiento del T. cruzi del líquido pericárdico se realizó un hemocultivo: una muestra de uno de los pacientes fue sembrada en medio NNN y medio LIT con SBF al $10 \%$ y mantenida a $25^{\circ} \mathrm{C}$ en el cepario del Laboratorio de Microbiología de la Universidad Industrial de Santander hasta la obtención de los parásitos para la prueba molecular.

\section{Estudio entomológico y de reservorios}

Inicialmente, la búsqueda activa de triatominos se abordó mediante la técnica de captura manual por hora/hombre en intradomicilio y peridomiclio y simultáneamente la aplicación de encuesta domiciliaria para determinar factores de riesgo en 58 viviendas del barrio Jerusalén. Luego, la búsqueda de vectores se realizó mediante la utilización de trampas Noireau 27 en el intra y peridomicilio. También se inspeccionaron árboles, montones de piedras y madera en los alrededores de las viviendas y se realizó la disección de 2 palmas de Attalea butyracea, cercanas a la vivienda del caso índice en el Jerusalén. Posteriormente, se amplió el rango de búsqueda de triatominos mediante el uso de trampas Angulo 28 en 49 palmas del área urbana del municipio de Aguachica. Las trampas, una por cada palma durante 12 horas entre las 18:00 y las 06:00, eran encajonadas en la axila de la primera bráctea de las palmas, cuya altura estaba entre 3,5 y 7,0 metros. Los insectos recolectados se identificaron hasta su especie, mediante la clave de Lent \& Wygodzinky ${ }^{29}$. Para la búsqueda de los reservorios se utilizó una trampa vivienda del vecindario del caso índice.

\section{Diagnóstico parasitológico de triatominos y reservorios}

A los triatominos capturados $-R$. pallescens- se les realizó un diagnóstico parasitológico para establecer la presencia de flagelados en las heces, según metodología descrita por Duque et al. 30 . Las heces positivas a flagelados de un triatomino $R$. pallescens se diluyeron en solución salina estéril a un $0,87 \%$ y se inocularon intraperitonealmente en dos ratones cepa ICR-CD1 que fueron mantenidos en el bioterio del Instituto Nacional de Salud.

Los reservorios fueron trampeados en las casas alrededor del caso índice, colocando secuencialmente una sola trampa Tomahawk por casa por noche.
Seguimiento del proceso infectivo en el modelo animal

Muestras sanguíneas de la parte terminal de la cola de los ratones inoculados con heces del triatomino positivas a flagelados fueron recolectadas semanalmente para establecer el desarrollo de su parasitemia y, después de 20 días, los ratones fueron sangrados vía intracardialmente para la siembra bajo condiciones de esterilidad. La cepa de Trypanosoma sp., aislada de esta forma, se mantuvo en el cepario a $25^{\circ} \mathrm{C}$ hasta la detección de formas parasitarias bajo microscopio invertido $40 \mathrm{X}$

\section{Estudios moleculares}

La cepa de T. cruzi en las deyecciones del vector (la misma que se inoculó en ratones ICR-CD1 y, posteriormente, se aisló en medios de cultivo Tobie y LIT) y aquella en el líquido pericárdico del paciente, se procesaron mediante la PCR, realizando una amplificación del ADN satélite de este parásito en el Laboratorio BCEI de la Universidad de Antioquia, siguiendo procedimientos descritos previamente 31 . Adicionalmente, las muestras fueron amplificadas con los iniciadores específicos para una región del gen miniexon que diferencia los subgrupos Ia, Ib y Id dentro del grupo TCI, según lo descrito por Falla et al. 32 .

Para determinar las preferencias alimenticias de los triatominos recolectados, una muestra de su contenido intestinal se amplificó con iniciadores específicos del gen citocromo b mitocondrial, mediante perfiles de alta resolución de fusión (HRM) por PCR en tiempo real, siguiendo protocolos previamente estandarizados para 14 reservorios 33 .

\section{Resultados}

Los resultados de los exámenes de laboratorio de las 11 personas involucradas en el brote aparecen en la tabla 2, donde se aprecia que sólo 4/11 pacientes presentaron parasitológicos directos positivos, mientras que $10 / 11$ resultaron positivos en los serológicos. Del hemocultivo de líquido pericárdico del paciente se logró el aislamiento de la una cepa de T. cruzi a las cuatro semanas. Por el contrario, los 53 hemocultivos de los contactos y convivientes de los casos confirmados resultaron todos negativos. 
Tabla 2

Exámenes de laboratorio de los 11 casos de enfermedad de Chagas aguda en Aguachica, Cesar, Colombia, 2010, procesados por el Laboratorio de Salud Pública de Santander, el Laboratorio de Salud Pública del Cesar y el Instituto Nacional de Salud.

\begin{tabular}{|c|c|c|c|c|c|}
\hline Paciente & Parentesco & Edad (años) & Directo & $\begin{array}{c}\text { ELISA IgG para } \\
\text { Chagas * }\end{array}$ & IFI \\
\hline A (caso inicial) & Caso índice & 20 & Negativo & $\begin{array}{c}\text { Positivo } \\
1,454\end{array}$ & $\begin{array}{c}\text { Reactivo } \\
1: 256\end{array}$ \\
\hline B (masculino) & Padre & 55 & $\begin{array}{c}\text { Micro hematocrito } \\
\text { Positivo }\end{array}$ & $\begin{array}{c}\text { Positivo } \\
1,237\end{array}$ & $\begin{array}{c}\text { Reactivo } \\
1: 256\end{array}$ \\
\hline$C$ (femenino) & Madre & 52 & $\begin{array}{c}\text { Micro hematocrito } \\
\text { Positivo }\end{array}$ & $\begin{array}{c}\text { 0,448 (resultado } \\
\text { borderline) }\end{array}$ & $\begin{array}{c}\text { Reactivo } \\
1: 256\end{array}$ \\
\hline$D$ (femenino) & Hermana & 25 & $\begin{array}{l}\text { Líquido pericárdico: } \\
\text { positivo para } \\
\text { Trypanosoma cruzi }\end{array}$ & $\begin{array}{l}\text { Positivo } \\
0,606\end{array}$ & $\begin{array}{c}\text { Reactivo } \\
1: 128\end{array}$ \\
\hline E (femenino) & Tía & 32 & $\begin{array}{l}\text { Micro hematocrito } \\
\text { Positivo }\end{array}$ & $\begin{array}{c}\text { Positivo } \\
1,624\end{array}$ & $\begin{array}{c}\text { Reactivo } \\
1: 128\end{array}$ \\
\hline F (femenino) & Abuela & 76 & Negativo & $\begin{array}{c}\text { Positivo } \\
0,626\end{array}$ & $\begin{array}{c}\text { Reactivo } \\
1: 256\end{array}$ \\
\hline G (femenino) & Tía & 45 & Negativo & $\begin{array}{c}\text { Positivo } \\
1,371\end{array}$ & $\begin{array}{c}\text { Reactivo } \\
1: 256\end{array}$ \\
\hline $\mathrm{H}$ (masculino) & Hermano & 24 & Negativo & $\begin{array}{c}\text { Positivo } \\
0,935\end{array}$ & $\begin{array}{c}\text { Reactivo } \\
1: 256\end{array}$ \\
\hline I (femenino) & Hermana & 18 & Negativo & $\begin{array}{c}\text { Positivo } \\
0,734\end{array}$ & $\begin{array}{l}\text { Reactivo } \\
1: 256 \text { ** }\end{array}$ \\
\hline $\mathrm{J}$ (femenino) & Sobrina & 7 & Negativo & $\begin{array}{l}\text { Positivo } \\
0,92 \text { ** }\end{array}$ & $\begin{array}{l}\text { Reactivo } \\
1: 64 \text { ** }\end{array}$ \\
\hline K (femenino) & Cuñada & 27 & Negativo & $\begin{array}{c}\text { Positivo } \\
\text { HIA 1:16 ** }\end{array}$ & $\begin{array}{l}\text { Reactivo } \\
1: 256 \text { ** }\end{array}$ \\
\hline
\end{tabular}

Nota 1: Laboratorio de Salud Pública Santander: ELISA punto de corte 0,45, técnica Elisa Omega. Se considera positivo valores por encima del punto de corte.

Nota 2: Laboratorio de Salud Pública del Cesar: ELISA punto de corte 0,529. Se considera positivo valores por encima del punto de corte;

Nota 3: Instituto Nacional de Salud: ELISA punto de corte 0,5. Se considera positivo valores por encima del punto de corte.

Nota 4: resultado de IFI se considera positivo títulos mayores o iguales a 1:32.

Nota 5: resultado de HIA se considera positivo títulos mayores o iguales a 1:16.

Fuente: Instituto Nacional de Salud 56.

\section{Estudio entomológico y de reservorios}

De acuerdo con la búsqueda de vectores y la encuesta domiciliaria no se evidenció domiciliación de triatominos. Sin embargo, se recolectaron 24 ninfas de $R$. pallescens en una palma de Ñoli (Elaeis oleifera) a 10m. de la vivienda de los casos. Con la especie predominante de $R$. pallescens (96\%) (24/25), en el barrio Jerusalén se recolectaron dos adultos de $P$. geniculatus y dos adultos y cinco ninfas de Eratyrus cuspidatus. El índice de infección con Trypanosoma sp. fue de un $54,2 \%$ (13/24) para R. pallescens. Al realizar la disección de las palmas en el barrio San José se hallaron dos zarigüeyas Didelphis marsupiales, una de las cuales resultó positiva para Trypano- soma sp. al diagnóstico parasitológico directo. El uso de la trampa Tomahawk no produjo ningún resultado positivo.

Según la encuesta domiciliaria, en el 58,6\% (34/58) de las viviendas los habitantes reconocen a los triatominos, de estos el $70,6 \%$ los conocen con el nombre de pitos; el $22,4 \%$ dicen verlos fuera de las viviendas y sólo el 5,2\% dice haberlos visto dentro de la vivienda. La caracterización de la vivienda mostró que en el 74,1\% (43/58) la construcción de la casa es de ladrillo, de estas sólo el $28 \%(12 / 43)$ se encuentran totalmente pañetadas; el 77,6\% (45/58) de las viviendas tienen suelo de cemento, y el 91,4\% (53/58) tienen techo de zinc. Se evidenció que el 44,8\% (26/58) de las viviendas tiene construcciones unidas o cerca- 
Distribución de animales domésticos en los anexos de la vivienda.

\begin{tabular}{lccccc} 
& Gallinero & Marranera & Tipo de anexo & & \\
& & & Conejera & $\begin{array}{c}\text { Gallinero y } \\
\text { marranera }\end{array}$ & $\begin{array}{c}\text { Gallinero, cocina, } \\
\text { lavadero }\end{array}$ \\
\hline$\%$ de uso & 19,0 & 3,4 & 1,7 & 10,3 & 1,7
\end{tabular}

Nota: la utilización de los anexos en las 21 viviendas encuestadas estuvo dominada por los gallineros y las marraneras.

nas a la casa principal, y en el $36,2 \%(21 / 58)$ de estas duermen animales domésticos como gallinas $(31 \%)(4 / 21)$ y cerdos $(3,4 \%)(1 / 21)$ (tabla 3$)$.

La utilización de trampas Angulo en las 49 palmas de vino (A. butyracea) arrojó un índice de infestación por triatominos del 30,6\% (15/49) con un total de 155 triatominos de R. pallescens, y un índice de infección por T. cruzi de $64 \%$ (71/111).

El estudio histopatológico con la cepa de $T$. cruzi, aislada a partir de triatomino, no mostró parasitemia en los órganos analizados y baja virulencia para el ratón, con gran dificultad para su adaptación y mantenimiento en estos animales de experimentación.

\section{Estudios moleculares}

Los análisis moleculares confirmaron la infección natural con T. cruzi I., tanto de $R$. pallescens, como en el paciente estudiado. Sin embargo, las muestras presentaron diferentes haplotipos de $T$. cruzi circulando en este municipio; en las heces del insecto $R$. pallescens se encontró el haplotipo TCIb, mientras que en la cepa aislada del paciente se encontró sólo el haplotipo TCIa (Figura 1). Los resultados de las pruebas moleculares para preferencias alimentarias en $R$. pallescens mostraron que los triatominos se alimentaron exclusivamente de gallinas, en ninguno se encontró sangre de humano.

\section{Discusión}

En el departamento del Cesar se han registrado ocho especies de triatominos: Belminus herreri, E. cuspidatus, P. geniculatus, Rhodnius neivai, R. pallescens, Rhodnius prolixus, T. dimidiata y Triatoma maculata 34 , mientras que para el municipio de Aguachica se tenía registro previo de $R$. pallescens, $P$. geniculatus y T. dimidiata. En 2010 Aguachica fue catalogada como de riesgo medio para la enfermedad de Chagas, según datos en la fase exploratoria del Programa Nacional de Pre- vención y Control de la Enfermedad de Chagas, realizado durante los años 1998 y 200135.

Ahora los resultados de este estudio evidencian la existencia de al menos dos ciclos naturales de transmisión de T. cruzi en este municipio en los que participan vectores silvestres, adaptados al microambiente de las palmas de $A$. butyracea $-R$. pallescens y P. geniculatus-, los cuales muy probablemente interactúan con animales sinantrópicos $-D$. marsupialis- alimentándose de ellos.

La presencia de grandes colonias de $R$. pallescens en estas palmas indican que estas plantas son el ecotopo natural de vectores infectados con T. cruzi en el área urbana del municipio de Aguachica, donde se evidenció que circulan al menos dos haplotipos de T. cruzi (TCIa, TCIb). Estudios de palmeras de A. butyracea en otras partes del país como: San Onofre, Sucre 36, Llanos Orientales y Valle del Magdalena 37, la región Momposina 38 indican que gracias a sus inflorescencias e infrutescencias albergan a varias clases de vertebrados e invertebrados, manteniendo de forma estable varios niveles tróficos del ecosistema 39 , estas características contribuyen a que sean un ecotopo importante para la trasmisión natural de T. cruzi, puesto que por sus condiciones climáticas permanecen durante todo el año infestadas de triatominos, especialmente $R$. pallescens.

Este estudio de los vectores y reservorios corrobora que el mecanismo de contaminación en el brote de enfermedad de Chagas agudo fue por transmisión oral y en el área urbana del municipio de Aguachica, al encontrarse al vector $R$. pallescens, un reservorio $D$. marsupialis en el extradomicilio infectado con TCI, al igual que el líquido pericárdico de un paciente. Los datos de este estudio también confirman que el linaje T. cruzi I predomina en Colombia, que especies silvestres de triatominos llegan hasta las viviendas y posibilitan, en eventos esporádicos por el mecanismo de contaminación oral, el ciclo de transmisión de la enfermedad de Chagas 40,41. 
Figura 1

Fotografías de los productos de la PCR de Trypanosoma cruzi en deyecciones de Rhodnius pallescens y líquido pericárdico de un paciente. Se distinguen claramente dos haplotipos que circulan en Aguachica, Cesar, Colombia.

1a) Cepa $\mathrm{TCl}$ de $R$. pallescens

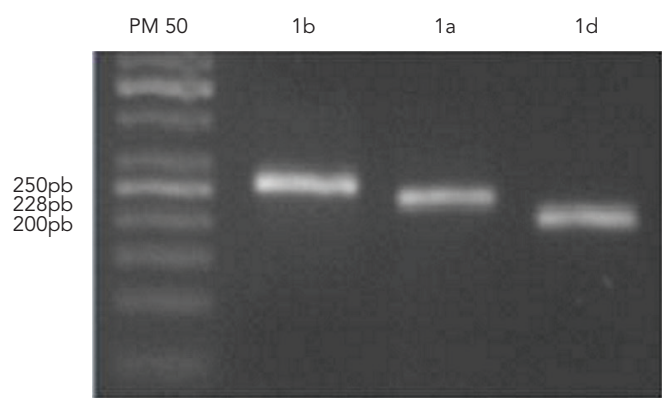

1b) Cepa TCla de humano DS

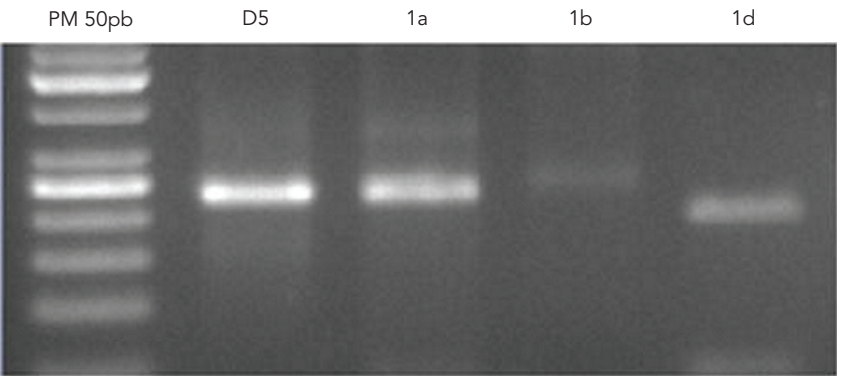

Estudios previos en la Amazonia brasileña revelaron que el TCI es el linaje más común en varias especies de mamíferos arbóreos, especialmente en Didelphis y con menor frecuencia en mamíferos terrestres y esporádicamente en mamíferos peridomésticos y humanos 42,43 .

Durante el estudio de campo no se encontraron triatominos adaptados al domicilio humano; sin embargo, la identificación de la sangre de gallina en el contenido intestinal de $R$. pallescences, muy probablemente procedente de las gallinas usadas como cebo en las trampas Angulo, indicaría un grado de aceptación por estos animales domésticos, ubicados en el peridomicilio de la vivienda humana, y aunque $R$. pallescens se considera una especie de hábitos estrictamente silvestres, podría ser atraída por la luz artificial de las viviendas y los gallineros ubicados en los peridomicilios para eventualmente alimentarse sobre estas aves ${ }^{44}$. De igual manera, la alta densidad de palmeras de la especie A. butyracea en el área urbana de Aguachica (49 palmas) y el porcentaje de infestación con triatominos de $R$. pallescens, infectados con T. cruzi, hacen factible las incursiones ocasionales de triatominos adultos en las viviendas, atraídos por la luz o en busca de alimentación sanguínea, con la probabilidad de contaminar algún alimento con sus deyecciones altamente infectadas con tripomastigotes metacíclicos. $R$. pallescens, siendo una especie de hábitos estrictamente silvestres, coloniza las viviendas y los peridomicilios, y según Jaramillo et al. ${ }^{44}$, esta especie puede estar incrementando su rol como vector en Colombia, pues como especie nativa que habita con frecuencia palmas, es capaz de invadir las habitaciones humanas lo que representa una tendencia al medio doméstico, aunque no sea una domesticación exitosa y permanente. Por lo tanto, en el municipio de Aguachica, catalogado como de riesgo medio para la transmisión de T. cruzi, dado que $R$. pallescens con frecuencia ha estado llegando a las viviendas junto con P. geniculatus y T. dimidiata, infectados naturalmente por T. cruzi, es importante mantener la vigilancia de los triatominos, en especial de $R$. pallescens, al cual se le puede conferir el estatus de candidato a vector primario más que de vector secundario 45 . Por otra parte, en el municipio de Aguachica, la presencia de poblaciones silvestres de $R$. pallescens en las palmeras que están cerca de las viviendas dificulta la efectividad de las actividades de control químico.

Es interesante descubrir que el riesgo aumenta con la deforestación indiscriminada, cuando se produce la migración de personas infectadas para la actividad de corte, y las barreras naturales que evitan la dispersión como son las palmeras (ecótopos de Rhodnius spp.) son quemadas y derribadas. Esta reducción de ecótopos (vegetación primaria y secundaria), donde se mantiene el ciclo silvestre de $T$. cruzi y la disminución de fuentes alimenticias para los triatominos, presiona a los triatominos a migrar en búsqueda de alimento, cada vez más cerca de los domicilios, donde son atraídos por la luz y ayudados por las corrientes de aire lo que hace factible la adaptación de triatominos silvestres al domicilio 46 .

Por otra parte, de acuerdo con la literatura, se considera que la estrategia de prevención 
para $R$. pallescens, especie silvestre con comportamientos de intrusión y posiblemente de colonización, pero sin domiciliación, debe basarse en la identificación de los focos silvestres de dispersión. La población humana afectada debe tener en cuenta que la marcada influencia de la fenología de las palmas 47 en la estacionalidad del ciclo de vida y la densidad poblacional de $R$. pallescens está asociada a condiciones climáticas regionales 36 .

En cuanto a P. geniculatus con amplia distribución geográfica en Latinoamérica, está asociada a nidos de aves y madrigueras de mamíferos en hábitat silvestres, generalmente de hábitos terrestres y subterráneos, puede ser encontrada eventualmente en el intradomicilio y peridomicilio, por lo que es considerado un vector de T. cruzi de importancia secundaria 29 . Por otro lado, por su baja tasa de crecimiento natural, baja tasa neta de reproducción, largo tiempo generacional y baja productividad reproductiva se considera poco probable que se convierta en una especie domiciliada de importancia significativa 48 , sin embargo, recientemente se ha incriminado como transmisor de $T$. cruzi en Mérida (Venezuela), estado no endémico para la patología chagásica 49; se ha encontrado en zonas urbanas de Caracas 50 y colonizando viviendas rurales al noreste de Antioquia 51. En el área de estudio podría ser el otro vector que se alimente sobre $D$. marsupialis y aunque sus poblaciones suelen tener bajas densidades presentan a menudo altos índices de infección con T. cruzi y al incursionar en la vivienda compiten con otros vectores por la alimentación sanguínea 52 y existe la posibilidad de que produzca contaminación de los alimentos 53 .

La captura de un D. marsupialis, infectado naturalmente con T. cruzi, confirma la participa- ción de estos reservorios sinantrópicos en un ciclo de transmisión. Estos marsupiales didélfidos, son referenciados, desde Carlos Chagas, y señalados como los más importantes reservorios del T. cruzi por las altas prevalencias de la infección natural por T. cruzi, relatada por diversos autores; su capacidad de mantener, en las glándulas odoríferas, el ciclo de multiplicación extracelular del parásito; su comportamiento nómada; y su capacidad de colonizar domicilio y peridomicilio en áreas forestales muy devastadas 54 .

Los marsupiales del género Didelphis son capaces de mantener simultáneamente los dos ciclos de multiplicación del T. cruzi, tanto en condiciones naturales como experimentales. De hecho, en las glándulas odoríferas, el parásito se puede multiplicar en la forma epimastigote y diferenciarse a tripomastigote metacíclico simultáneamente al ciclo multiplicativo intracelular de los diversos tejidos del animal. Eso significa que la zarigüeya puede, al mismo tiempo, ser reservorio y vector del T. cruzi. Estas características exige que estos animales deban ser objeto de vigilancia epidemiológica, puesto que son, pueden participar de más de un ciclo de transmisión del T. cruzi en el área.

\section{Conclusión}

Triatominos silvestres como: $R$. pallescens y $P$. geniculatus en ecotopos naturales -palmas de $A$. butyracea y E. oleifera-dentro o en la periferia de comunidades rurales tienen ahora mayor participación en la trasmisión de T. cruzi oral, pues por intrusiones esporádicas al domicilio del hombre pueden contaminar sus alimentos y bebidas para provocar brotes de Chagas agudo, afectando a todos los miembros de una familia. 


\section{Resumo}

A Colômbia tem registro de 11 surtos da doença de Chagas e 80 casos agudos por contaminação oral com Trypanosoma cruzi. Esta pesquisa analisa os achados entomológicos e parasitológicos do surto de Aguachica, Cesar, em 2010. Um grupo interdisciplinar de profissionais da saúde e de universidades regionais efetuou os testes laboratoriais nos pacientes e o estudo de foco de transmissão. Encontraram 11 casos agudos da doença de Chagas em uma única família com domicilio sem triatomíneos e Rhodnius pallescens, Pantrongylus geniculatus, Eratyrus cuspidatus e dois Didelphis marsupialis infectados com $\mathrm{T}$. cruzi em palmeiras de Attalea butyracea $e$ Elaeis oleifera da área urbana de Aguachica. Analisa-se a participação de R. pallescens e a função das palmeiras em o ciclo silvestre de T. cruzi e para a transmissão oral da doença de Chagas. Incursões esporádicas de R. pallescens, P. geniculatus $e \mathrm{E}$. cuspidatus silvestres das palmeiras próximas ao domicilio humano podem provocar surtos cada vez mais frequentes de Chagas oral.

Doença de Chagas; Trypanosoma cruzi; Surto de Doenças

\section{Colaboradores}

H. Soto contribuyó con el diseño del estudio, adquisición de datos y revisión del manuscrito. T. Tibaduiza contribuyó con la elaboración del manuscrito, adquisición de datos y análisis de la información. M. Montilla contribuyó con la adquisición, análisis e interpretación delos datos y revisión. O. Triana contribuyó con la adquisición, análisis e interpretación de los datos y revisión. D. C. Suárez contribuyó con adquisición, análisis e interpretación de los datos, revisión. M. T. Torres contribuyó con análisis e interpretación de los datos, elaboración y revisión del manuscrito. M. T. Arias contribuyó con el diseño del estudio, adquisición de datos y revisión del manuscrito. L. Lugo contribuyó con el diseño del estudio y revisión del manuscrito.

\section{Agradecimientos}

Los autores agradecen a los habitantes de Aguachica por su colaboración durante la realización de la fase de trabajo de campo.

\section{Referencias}

1. Guhl F, Restrepo M, Angulo VM, Antunes CM, Campbell-Lendrum D, Davies CR. Lessons from a national survey of Chagas disease transmission risk in Colombia. Trends Parasitol 2005; 21:259-62.

2. United Nations Development Program; World Bank; WHO Special Program for Research and Training in Tropical Disease. Thirteenth program report. Geneva: World Health Organization; 1997.

3. Moncayo A, Silva AC. Current trends and future prospects for control of Chagas Diseases. In: Telleria J, Tibayrenc M, editors. American trypanosomiasis (Chagas disease): one hundred years of the research. London: Elsevier; 2010. p. 55-82.

4. World Health Organization. Control of Chagas disease. Second report of the Expert Committee. Geneva: World Health Organization; 2002. (WHO Technical Report Series, 905).

5. Silveira AC. New challenges and the future of control. Rev Soc Bras Med Trop 2011; 44 Suppl 2:122-4.

6. World Health Organization. Control of Chagas disease. Geneva: World Health Organization; 2002. (Technical Report Series, 905).

7. Dujardin JP, Schofield CJ, Tibayrenc M. Population structure of Andean Triatoma infestans: allozyme frequencies and their epidemiolgoical relevance. Med Vet Entomol 1988; 12:20-9.
8. Shikanai-Yasuda MA, Carvalho NB. Oral transmission of Chagas disease. Clin Infect Dis 2012; 54:845-52.

9. Coura JR, Junqueria A, Fernandes O, Valente A, Miles MA. Emerging Chagas disease in the Amazonian Brazil. Trends Parasitol 2002; 18:171-6.

10. Dias JCP. A doenca de Chagas como problema do continente americano. http://www.fiocruz. $\mathrm{br} / \mathrm{chagas} / \mathrm{cgi} / \mathrm{cgilua}$.exe/sys/start.htm?sid=134 (accedido el 15/Ago/2010).

11. Pinto AYN, Valente SA, Valente VC, Ferreira Junior AG, Coura JR. Fase aguda da doença de Chagas na Amazônia brasileira. Estudo de 233 casos do Pará, Amapá e Maranhão observados entre 1988 e 2005. Rev Soc Bras Med Trop 2008; 41:602-14.

12. Nóbrega AA, García MH, Tatto E, Obara MT, Costa E, Sobel J, et al. Oral transmission of Chagas disease by consumption of açaí palm fruit, Brazil. Emerg Infect Dis 2009; 15:653-5.

13. Cardoso AVN, Lescano SAZ, Neto VA, Gakiya E, Santos SV. Avaliação da sobrevida de Trypanosoma cruzi em cana de açúcar utilizada no preparo do caldo. Rev Inst Med Trop São Paulo 2006; 48:287-9.

14. Silveira AC, Dias JCP. The control of vectorial transmission. Rev Soc Bras Med Trop 2011; 4:52-63. 
15. Añez N, Crisante G, Romero M. Supervivencia e infectividad de formas metacíclicas de Trypanosoma cruzi en alimentos experimentalmente contaminados. Bol Malariol Salud Ambient 2009; 49:91-6.

16. Dias JCP. Doença de Chagas no seculo XXI. http:// www.fac.org.ar/cvirtual/cvirtesp/cientesp/ecesp/ ecc4500c/cpinto/cpinto.htm (accedido el 20/ Ago/2010).

17. Suárez DC, Rey AP, Ordúz ML, Prada RL, Tarazona Z. Supervivencia de Trypanosoma cruzi en bebidas experimentalmente contaminadas. Biomédica (Bogotá) 2012; 32:134-8.

18. Organización Panamericana de la Salud. Enfermedad de Chagas. Guía para vigilancia, prevención, control y manejo clínico de la enfermedad de Chagas aguda transmitida por alimentos. Río de Janeiro: Centro Panamericano de Fiebre Aftosa, Organización Panamericana de la Salud; 2009.

19. Nicholls RS. Enfermedad de Chagas como enfermedad transmitida por alimentos: la experiencia en Colombia. In: Unidad Regional de Prevención y Control de Enfermedades Transmisibles, Grupo Técnico Especializado en Inocuidad de Alimentos, Organización Panamericana de la Salud. Informe final consulta técnica en epidemiología, prevención y manejo de la transmisión de la enfermedad de Chagas como enfermedad transmitida por alimentos (ETA). Río de Janeiro: Organización Panamericana de la Salud/Organización Mundial de la Salud; 2006. p. 13-4.

20. Hernández LM, Ramírez AN, Cucunubá Z, Zambrano P. Brote de Chagas agudo en Lebrija, Santander 2008. Revista del Observatorio de Salud Pública de Santander 2009; 4:28-36.

21. Montilla MM, Gutiérrez M, Parra E, Bueno A, Torres M, Esteban L, et al. Pesquisas entomológicas y parasitológicas de brotes de Chagas agudo en el área metropolitana de Bucaramanga, departamento de Santander, Colombia. Infectio 2012; 16(1S):82.

22. Departamento Nacional de Estadística. Censo nacional. http://www.dane.gov.co/index.php/ poblacion-y-registros-vitales/censos/censo-2005 (accedido el 23/Sep/2010).

23. Holdrige LR. Determinación of world plant formation from simple climate data. Science 1947; 105(2727):367-8.

24. Espinal S, Montenegro E. Formaciones vegetales de Colombia y mapa ecológico. (1:1.000.000). Bogotá: Instituto Geográfico Agustín Codazzi; 1963

25. Murphy PG, Lugo AE. Ecology of tropical dry forest. Annu Rev Ecol System 1986; 17:67-8.

26. Guhl F, Nicholls RS. Manual de procedimientos para el diagnóstico de la enfermedad de Chagas. Bogotá: Quebecor Impresores; 2001.

27. Noireau F, Abad-Franch F, Valente SAS, Dias-Lima A, Lopes CM, Cunha V, et al. Trapping Triatominae in silvatic habitats. Mem Inst Oswaldo Cruz 2002; 97:61-3.

28. Angulo VM, Esteban L. Nueva trampa para la captura de triatominos en hábitats silvestres y peridomésticos. Biomédica (Bogotá) 2011; 31:364-8.

29. Lent H, Wygodzinsky P. Revision of the Triatominae (Hemiptera, Reduviidae) and their significance as vectors of Chagas disease. Bull Am Mus Nat Hist 1979; 163:123-520.
30. Duque S, Peláez D, Gualdrón LE, Villarreal E, Corredor-Arjona A. Aislamiento de tripanosomas a partir de materia fecal de Rhodnius prolixus. Biomédica (Bogotá) 1988; 8:37-9.

31. Souto RP, Fernandes O, Macedo AM, Campbell DA, Zingales B. DNA markers define two major phylogenetic lineages of Trypanosoma cruzi. Mol Biochem Parasitol 1996; 83:141-52.

32. Falla A, Herrera C, Fajardo A, Montilla M, Vallejo G, Gulh F. Haplotype identification within Trypanosoma cruzi I in Colombian isolates from several reservoirs, vectors and humans. Acta Trop 2009; 11:15-21.

33. Peña VH, Fernández GJ, Gómez-Palacio AM, Mejía-Jaramillo AM, Cantillo O, Triana-Chávez O. High-resolution melting (HRM) of the cytochrome B gene: a powerful approach to identify bloodmeal sources in Chagas disease vectors. PLoS Negl Trop Dis 2012; 6:153.

34. Guhl F, Aguilera G, Pinto N, Vergara D. Actualización de la distribución geográfica y ecoepidemiología de la fauna de triatominos (Reduviidae: Triatominae) en Colombia. Biomédica (Bogotá) 2007; 27 Suppl 1:143-62.

35. Gioiavanetti M, Lomanto J, Soto H. Situación de la enfermedad de Chagas en Colombia. In: Guhl F, editor. Primer taller internacional sobre control de la enfermedad de Chagas. Curso de diagnóstico, manejo y tratamiento de la enfermedad Chagas. Sexta reunión de la iniciativa para el control de la enfermedad de Chagas. Bogotá: Ediciones Uniandes; 2005. p. 173-87.

36. Pizarro JC, Romaña C. Variación estacional de una población silvestre de Rhodnius pallescens Barber 1932 (Heteroptera: Triatomine ) en la costa caribe colombiana. Bulletin de l'IFEA 1998; 27:309-25.

37. Vallejo GA, Lozano LE, Carranza JC, Sánchez JL, Jaramillo JC, Guhl F, et al. Ecología de los triatominos no domiciliados en Colombia con especial referencia a Rhodnius colombiensis en el departamento del Tolima. In: Vallejo GA, Carranza MJC, Jaramillo JC, editores. Biología, epidemiología y control de la tripanosomiasis americana y leishmaniosis. Ibagué: Laboratorio de Investigaciones en Parasitología Tropical, Universidad del Tolima; 2000. p. 22-8.

38. Salazar DA, Calle J. Caracterización ecoepidemiológica de Rhodnius pallescens en la palma Attalea butyracea en la Región Momposina (Colombia). Actualidades Biológicas 2003; 25:31-8.

39. Teixeira ARL, Monteiro PS, Rebelo JM, Argañaraz ER, Vieira D, Lauria-Pires L, et al. Emerging Chagas disease: trophic network and cycle of transmision of Trypanosoma cruzi from palm trees in the Amazon. Emerg Infect Dis 2001; 7:100-12.

40. Monteiro WM, Barbosa MGV, Toledo MJO, Fé FA, Fé NF. Série de casos agudos de doença de Chagas atendidos num serviço terciário de Manaus, Estado do Amazonas, de 1980 a 2006. Rev Soc Bras Med Trop 2010; 43:207-10.

41. Reyes M, Esteban L, Torres FA, Flórez M, Agudelo JC, Angulo VM. Intrusión de Pastrongylus geniculatus y Rhodnius pallescens a viviendas y áreas sociales en un barrio de Bucaramanga, Santander, Colombia. Biomédica (Bogotá) 2011; 31 Suppl 3:54 
42. Marcili A, Valente VC, Valente SA, Junqueira AC, da Silva FM, Pinto AY, et al. Trypanosoma cruzi in Brazilian Amazonia: lineages TCI and TCIIa in wild primates, Rhodnius spp. and in humans with Chagas disease associated with oral transmission. Int J Parasitol 2009; 39:615-23.

43. Añez N, Crisante G, Romero M. Supervivencia e infectividad de formas metacíclicas de Trypanosoma cruzi en alimentos experimentalmente contaminados. Bol Malariol Salud Ambient 2009; 49:91-6.

44. Jaramillo N, Schofield CJ, Gorla D, Caro-Riaño H, Moreno J, Mejia E, et al. The role of Rhodnius pallescens as a vector of Chagas disease in Colombia and Panamá. Research and Reviews in Parasitology 2000; 60:75-82.

45. Guhl F, Pinto N, Aguilera G. Sylvatic triatominae: a new challenge in vector control transmission. Mem Inst Oswaldo Cruz 2009; 104 Suppl I:71-5.

46. Gottdenker NL, Calzada JE, Saldaña A, Carroll CR. Association of anthropogenic land use change and increased abundance of the Chagas disease vector Rhodnius pallescens in a rural landscape of Panama. Am J Trop Med Hyg 2011; 84:70-7.

47. Rioux JA, Dereure J, Pereieres J. Approche écologique du risque epidémiologioque. L'exemple des leishmanioses. Bulletin d'Ecologie 1990; 21:1-9.

48. Cabello DR, Galíndez Girón I. Vital statistics of Panstrongylus geniculatus (Latreille, 1811) (Hemiptera: Reduviidae) under experimental conditions. Mem Inst Oswaldo Cruz 1998; 93:257-62.

49. Añéz N, Saavedra C, Crisante G, Rojas A, Lizano E. Infección natural por Trypanosoma cruzi en Panstrongylus geniculatus (Latreille, 1811) de la región montana de Mérida, Venezuela. Bol Malariol Salud Ambient 2005; 45:139-41.
50. Pifano F. El potencial enzoótico silvestre del complejo ecológico Schizotrypanum cruzi - Didelphis marsupialis - Panstrongylus geniculatus y sus incursiones a la vivienda humana del Valle de Caracas, Venezuela. Boletín de la Academia de Ciencias Físicas, Matemáticas y Naturales 1986; XLVI:9-37.

51. Wolff M, Castillo D. Evidencias de domesticación y aspectos biológicos de Panstrongylus geniculatus (Latreille, 1811) (Hemiptera: Reduviidae). Acta Entomológica Chilena 2000; 24:77-83.

52. Feliciangeli MD, Carrasco H, Patterson JS, Suarez B, Martínez C, Medina M. Mixed domestic infestation by Rhodnius prolixus Stäl, 1859 and Panstrongylus geniculatus Latreille, 1811, vector incrimination, and seroprevalence for Trypanosoma cruzi among inhabitants in El Guamito, Lara State, Venezuela. Am J Trop Med Hyg 2004; 71:501-5.

53. Alarcón de Noya B, Díaz-Bello Z, Colmenares C, Ruiz-Guevara R, Mauriello L, Zavala-Jaspe R, et al. Large urban outbreak of orally acquired acute Chagas disease at a school in Caracas, Venezuela. J Infect Dis 2010; 201:1308-15.

54. Deane M. Trypanosoma cruzi vertebrate and invertebrate cycles in the same mammal host, the opossum Didelphis marsupiales. Mem Inst Oswaldo Cruz 1984; 79:513-5.

55. Subdirección de Vigilancia y Control de Salud Pública, Instituto Nacional de Salud. Informe Epidemiológico de Santander 2010; 4(1).

56. Subdirección de Vigilancia y Control de Salud Pública, Instituto Nacional de Salud. Situación brote Chagas agudo de posible transmisión oral en Aguachica, Cesar. Informe de Avance 2010; (6).

Recibido el 08/Feb/2013

Versión final presentada el 25/Jul/2013

Aprobado el 04/Oct/2013 